\title{
Uncovering deterministic causal structures: a Boolean approach
}

\author{
Michael Baumgartner
}

Received: 10 November 2007 / Accepted: 16 May 2008 / Published online: 11 June 2008

(C) Springer Science+Business Media B.V. 2008

\begin{abstract}
While standard procedures of causal reasoning as procedures analyzing causal Bayesian networks are custom-built for (non-deterministic) probabilistic structures, this paper introduces a Boolean procedure that uncovers deterministic causal structures. Contrary to existing Boolean methodologies, the procedure advanced here successfully analyzes structures of arbitrary complexity. It roughly involves three parts: first, deterministic dependencies are identified in the data; second, these dependencies are suitably minimalized in order to eliminate redundancies; and third, one or-in case of ambiguities - more than one causal structure is assigned to the minimalized deterministic dependencies.
\end{abstract}

Keywords Causation - Causal reasoning - Discovery algorithms ·

Deterministic structures

\section{Introduction}

Since the early nineties, the philosophical literature on causal reasoning has been dominated by inference procedures developed within a theoretical framework according to which causal structures can be analyzed in terms of Bayesian networks. ${ }^{1}$ One of the key studies that has influenced and structured that whole research program, undoubtedly, is Spirtes', Glymour's and Scheines' book on Causation, Prediction, and Search (2000). That study has inspired and provoked a host of literature that has in the mean time profoundly deepened our understanding of how, under what conditions, and to what extent causal structures can be inferred from pertinent empirical

$\overline{1}$ With respect to the notion of a Bayesian network cf. e.g. Pearl (1985).

M. Baumgartner $(\bowtie)$

University of Bern, Bern, Switzerland

e-mail: baumgartner@philo.unibe.ch 
data. As is well known, all the different causal discovery algorithms developed in that framework impose two important constraints on the causal structures and the data generated by these structures: The structures and the data must satisfy the causal Markov assumption and the faithfulness assumption. ${ }^{2}$

Many causal structures undoubtedly satisfy these assumptions, but certain common structures do not. One important type of causal structure that does not conform to both of these assumptions is constituted by deterministic structures that are investigated on a sufficiently fine-grained level such that deterministic dependencies actually show up in the data. In a deterministic structure every value of at least one exogenous variable uniquely determines the values of at least one endogenous variable. ${ }^{3}$ Such deterministic dependencies may, of course, not show up in corresponding data, if, for instance, not all variables involved in the structure are contained in the set of investigated variables or if not all relevant factors are controlled for in a respective study. However, if deterministic structures are investigated against a causally homogeneous background-say, in a laboratory context — or if only few instances of a causal structure are available — as in small- $N$ studies in social sciences ${ }^{4}$ — to the effect that deterministic dependencies are actually exhibited in the data, the faithfulness assumption is violated and, accordingly, standard procedures for the discovery of causal Bayesian networks are not applicable or generate inadequate outputs, respectively. ${ }^{5}$

This paper introduces a procedure of causal reasoning that is custom-built for deterministic structures and deterministic data. As the procedure analyzes so-called coincidence data, which is going to be properly characterized in subsequent sections, it shall be labeled coincidence analysis, or CNA for short. ${ }^{6}$ While procedures uncovering causal Bayes nets explicitly or implicitly presuppose a probabilistic notion of causation, CNA draws on an account of causation that is inspired by the regularity theoretic tradition going back to Mackie's (1974) theory of INUS-conditions. Instead of Bayesian networks, CNA implements Boolean techniques, predecessors of which can be found in Quine (1952; 1959), Ragin (1987; 2000) and May (1996; 1999). In a nutshell, the procedure consists of three parts: In a first step deterministic dependencies of sufficiency and necessity are identified in the data; a second step suitably minimalizes these dependencies in order to eliminate redundancies; and in a third step the minimalized dependencies are causally interpreted. Before CNA is properly

\footnotetext{
2 The causal Markov assumption states that in a probability distribution $\mathcal{P}$ generated by a (acyclic) causal structure $\mathcal{S}$ a variable $Z$ is independent of all its non-effects in $\mathcal{S}$ conditional on all of $Z$ 's direct causes, provided that no direct common causes of any two variables in $\mathcal{S}$ are left out of $\mathcal{P}$. According to the faithfulness assumption, there are no other conditional independence relations in $\mathcal{P}$ than the ones implied by the causal Markov assumption (cf. e.g. Spirtes etal. 2000, pp. 29-31; Glymour 1997, 2007).

3 For details cf. Glymour (2007, p. 236).

4 Cf. e.g. Ragin (1987).

5 Such as to illustrate violations of faithfulness by deterministic structures assume that a factor $B$ is a common cause of $A$ and $C$, i.e. $A \longleftarrow B \longrightarrow C$, and that $B$ is sufficient and necessary for $C$. In that case, $B$ and $A$ are independent conditional on $C$, i.e. $p(A \mid B \wedge C)=p(A \mid C)$, which is not implied by the causal Markov assumption (cf. e.g. Spirtes et al. 2000, pp. 53-57; Glymour 2007).

6 Coincidence analysis is not abbreviated by "CA" because, in the social science literature, this acronym is often used for correspondence analysis which must not be confused with coincidence analysis.
} 
introduced and illustrated in Sects. 3-9, Sect. 2 is going to briefly review its conceptual background.

\section{Conceptual background}

As mentioned above, the conceptual background of the inference procedure to be developed in this paper is located in the regularity theoretic tradition of the philosophy of causation. According to one of the core tenets of regularity theories, causation is deterministic. The question as to whether all causal processes indeed are ultimately deterministic or not can be bypassed here. It seems hardly questionable that there are at least some deterministic processes-especially at a macro level. Thus, whoever holds that there additionally exist irreducibly indeterministic causal processes, e.g. at a micro level, can simply view regularity theories as being concerned with the deterministic structures at a macro level only. However, as is well known, the adequacy of regularity theoretic analyses of deterministic causal structures has been broadly criticized during the past 30 years. While I have argued elsewhere (cf. Baumgartner 2008a) that that criticism has commonly targeted oversimplified regularity theoretic sketches, this is not the place to defend a sufficiently sophisticated regularity theory of causation. The prospects and merits of CNA, in the end, do not hinge on whether deterministic causal structures can successfully be reduced to regularities subsisting in nature or not. All that matters for my current purposes is that when it comes to deterministic structures regularities undoubtedly constitute an important sort of empirical information on which inferences to underlying structures can be based.

CNA is designed to unfold deterministic causal structures on type level, i.e. it analyzes general causation. The relata of general causation can be seen to be event types or factors for short. A factor that causes another factor is said to be causally relevant to the latter. Factors are taken to be similarity sets of event tokens. They are sets of type identical token events, of events that share at least one feature. Whenever a member of a similarity set that corresponds to an event type occurs, the latter is said to be instantiated. Factors are symbolized by italicized capital letters $A, B, C$, etc., with variables $Z, Z_{1}, Z_{2}$ etc. running over the domain of factors. They are negatable. The negation of a factor $A$ is written thus: $\bar{A} \cdot \bar{A}$ is simply defined as the complementary set of $A$. Alternatively, factors can be seen as binary variables that take the value 1 whenever an event of the corresponding type occurs and the value 0 whenever no such event occurs. That means CNA is custom-built for deterministic structures featuring binary variables. The restriction to binary variables primarily serves conceptual simplicity. It allows for a straightforward implementation of Boolean optimization techniques, which shall turn out to be of great relevance to the uncovering of deterministic structures. Nonetheless, the restriction to binary variables implies that structures involving multi-valued variables must be encoded in binary terms before they can be treated by CNA. For quite some time, however, there have been considerable efforts in the literature on logic synthesis to generalize Boolean optimization procedures as Quine-McCluskey optimization for systems involving multi-valued variables (cf. e.g. Mirsalehi and Gaylord 1986 or Sasao 1999, Chap. 10). Even though-as we shall see in Sect.9-CNA significantly differs from the Quine-McCluskey algorithm, there 
seem to be no principled obstacles to generalizing CNA for multi-valued variables as well. That, however, is not going to be attempted in the present context. For reasons of simplicity, CNA shall here be tailored to the case of causal structures involving binary variables, i.e. factors, only.

Causal analyses are always relativized to a set of investigated factors. This set is referred to as the factor frame of the analysis. Factors are virtually never causally relevant to their effects in isolation. Rather, they are parts of whole causing complexes-complex causes. A complex cause only becomes causally effective if all of its constituents are co-instantiated, i.e. instantiated close-by or coincidently. Coincidently instantiated factors are termed coincidences. As will be shown below, coincidences constitute the empirical data processed by CNA.

Essentially, modern regularity theories analyze causal relevance with recourse to minimalized regularities among factors. The crucial notion needed in the definiens of causal relevance is the notion of a minimal theory. ${ }^{7}$ Briefly, a minimal theory of a factor $B$ is a minimally necessary disjunction of minimally sufficient conditions of $B$. A conjunction of coincidently instantiated factors $A_{1} \wedge A_{2} \wedge \cdots \wedge A_{n}$, which for simplicity shall be abbreviated by a mere concatenation of the respective factors, is a minimally sufficient condition of a factor $B$ iff $A_{1} A_{2} \ldots A_{n}$ is sufficient for $B$, i.e. $A_{1} A_{2} \ldots A_{n} \rightarrow B$, and there is no proper part $\alpha$ of $A_{1} A_{2} \ldots A_{n}$ such that $\alpha \rightarrow B$. A "proper part" of a conjunction designates the result of any reduction of this conjunction by one conjunct. ${ }^{8}$ Analogously, a disjunction of factors $A_{1} \vee A_{2} \vee \cdots \vee A_{n}$ is a minimally necessary condition of a factor $B$ iff $A_{1} \vee A_{2} \vee \cdots \vee A_{n}$ is necessary for $B$, i.e. $B \rightarrow A_{1} \vee A_{2} \vee \cdots \vee A_{n}$, and there is no proper part $\beta$ of $A_{1} \vee A_{2} \vee \cdots \vee A_{n}$ such that $B \rightarrow \beta$. A "proper part" of a disjunction designates the result of any reduction of this disjunction by one disjunct.

That a disjunction of minimally sufficient conditions of a factor $B$ is minimally necessary for $B$ shall be symbolized by ' $\Rightarrow$ ' which is termed a double-conditional. Thus, a minimal theory has the following double-conditional form:

\section{(1) $A C \vee D E \vee F G H \Rightarrow B$}

Informally, (1) says that whenever $A C$ or $D E$ or $F G H$ are instantiated, $B$ is instantiated as well, and that whenever $B$ is instantiated $A C$ or $D E$ or $F G H$ is instantiated as well, and that sufficient and necessary conditions contained in (1) are minimal. In this vein, both the principle of determinism and the principle of causality are formally captured in a straightforward way: Causes determine their effects, and if no causes are present, the effect is not present either. Membership in a minimal theory induces positive direct causal relevance: A factor $A$ is (positively) directly causally relevant

\footnotetext{
7 Cf. e.g. Graßhoff and May (2001), Baumgartner and Graßhoff (2004) or Baumgartner (2008a).

8 Defining a minimally sufficient condition in terms of proper parts and not —as might be expected-in terms of proper subsets that correspond to reductions of sufficient conditions by one or more conjuncts allows for a simpler procedure to identify minimally sufficient conditions. For if a sufficient condition has no sufficient proper parts, it does not have sufficient proper subsets either. Hence, in order to show that a sufficient condition $A_{1} A_{2} \ldots A_{n}$ is minimally sufficient it suffices to establish that $A_{1} A_{2} \ldots A_{n}$ has no proper parts-establishing that it has no sufficient proper subsets is unnecessary. For analogous reasons minimally necessary conditions are defined in terms of proper parts and not proper subsets in the present context.
} 
to a factor $B$ iff $A$ is part of a minimal theory of $B .{ }^{9}$ Hence, (1) represents a causal structure such that $A C, D E$, and $F G H$ are alternative complex causes of $B$. Correspondingly, a factor $A$ has negative direct causal relevance for a factor $B$ iff $\bar{A}$ is contained in a minimal theory of $B$.

Analyzing the disjunction of alternative deterministic causes of $B$ as a necessary condition of $B$ amounts to claiming sufficiency of $B$ for just that disjunction. As is often done by critics of regularity accounts, the question might thus be raised as to how the above account of causal relevance captures the undisputed non-symmetry of that relation. ${ }^{10}$ For if $B$ can be shown to be minimally sufficient for $A C \vee D E \vee F G H$, it might be argued that - relative to the above analysis $-B$ is likewise to be considered causally relevant to its alternative causes. Contrary to first appearances, however, double-conditionals as (1) are not symmetrical with respect to the expressions to the left and the right of " $\Rightarrow$ ". The instantiation of a particular disjunct is minimally sufficient for $B$, but not vice versa. $B$ does not determine a particular disjunct to be instantiated. ${ }^{11} B$ only determines the whole disjunction of minimally sufficient conditions. $A C$ and $D E$ and $F G H$ are each minimally sufficient for $B$, the latter however is only minimally sufficient for $A C \vee D E \vee F G H$. This non-symmetry corresponds to the direction of determination.

Accounting for the non-symmetry of causal relevance in this vein has an important implication as regards the minimal complexity of deterministic structures. A condition $A C$ that is both minimally sufficient and necessary for a factor $B$ cannot be identified as the cause of $B$, for, in that case, $B$ is minimally sufficient and necessary for $A C$ as well. All empirical evidence such mutual dependencies generate are perfectly correlated instantiations of $A C$ and $B$-both are either co-instantiated or absent. Such data can either stem from a structure such that $A C$ is a cause of $B$ or vice versa or $A C$ and $B$ are parallel effects of an uncontrolled hidden cause. If no additional empirical information such as temporal orderings of the instances of $A C$ and $B$ is available, neither $A C$ nor $B$ can be identified as cause or effect. As is well known, similar ambiguities arise in case of probabilistic data analyzed by procedures uncovering causal Bayes nets (cf. Spirtes etal. 2000). Causes and effects can only be kept apart based on regularity or correlation data alone if the data is diverse enough such that at least two alternative causes of each effect are contained in the corresponding factor frame. Section 9 will be concerned in detail with ambiguities that arise when it comes to causally analyzing data featuring deterministic dependencies.

\footnotetext{
9 In fact, in order for a minimal theory $\Phi$ to be causally interpretable, certain relational constraints, as spatiotemporal proximity, have to be imposed on the events that instantiate the factors in $\Phi$. For simplicity, these constraints are neglected here. For a detailed presentation of the logical form of minimal theories cf. Baumgartner (2008a). Furthermore, as shall become apparent in Sect. 7 below, minimalizing necessary conditions paves the way for an accurate regularity theoretic treatment of common cause structures, which—on account of Mackie's (1974) famous Manchester Factory Hooters counterexample_-have often been considered intractable by regularity theories.

10 For details on the notion of non-symmetry cf. e.g. Lemmon (1965, p. 180). The relation of general causation, which is of interest in the present context, is non-symmetric and not asymmetric as is often claimed in the literature. Generic causal dependencies may be cyclically structured.

11 Cf. Graßhoff and May (2001, pp. 97-99) and Baumgartner (2008a). Similar analyses of the direction of causation have been proposed in Sanford (1976), Ehring (1982), and Hausman (1998).
} 
Ordinary causal structures far exceed (1) in complexity. Most causally relevant factors are of no interest to causal investigations or are unknown. That is why minimal theories either need to be relativized to a specific causal background or must be kept open for later extensions. The latter is achieved by means of variables. Variables $X_{1}, X_{2}, \ldots$ are introduced to stand for an open (finite) number of additional conjuncts within a sufficient condition, while $Y_{A}, Y_{B}, \ldots$ are taken to stand for an open number of additional disjuncts in a minimal theory. If (1) is in this sense kept open for additional factors, one gets:

\section{(2) $A C X_{1} \vee D E X_{2} \vee F G H X_{3} \vee Y_{B} \Rightarrow B$}

While direct causal relevance is analyzed with recourse to membership in simple minimal theories as (1) or (2), complex causal structures as causal chains or common cause structures are represented by complex minimal theories. Simple minimal theories can be conjunctively concatenated to complex theories: A conjunction of two minimal theories $\Phi$ and $\Psi$ is a complex minimal theory iff, first, at least one factor in $\Phi$ is part of $\Psi$ and, second, $\Phi$ and $\Psi$ do not have an identical consequent. The first constraint guarantees that complex theories represent cohering causal structures and the second restriction prohibits the conjunctive concatenation of equivalent minimal theories and thus excludes redundancies. The following are two examples of complex minimal theories:

(3) $\left(A X_{1} \vee D X_{2} \vee Y_{B} \Rightarrow B\right) \wedge\left(B X_{3} \vee G X_{4} \vee Y_{H} \Rightarrow H\right)$

(4) $\left(A X_{1} \vee D X_{2} \vee Y_{B} \Rightarrow B\right) \wedge\left(D X_{3} \vee G X_{4} \vee Y_{H} \Rightarrow H\right)$

(3) represents a causal chain- $B$ is the effect factor of the first conjunct and a cause factor in the second conjunct, (4) stands for a common cause structure- $D$ is a common cause of $B$ and $H$. In this vein, deterministic causal structures of arbitrary complexity can be represented on regularity theoretic grounds. Accordingly, a factor $A$ can be said to be indirectly causally relevant to a factor $B$ iff there is a sequence of factors $Z_{1}, Z_{2}, \ldots, Z_{n}, n \geq 3$, such that $A=Z_{1}, B=Z_{n}$, and for each $i, 1 \leq i<n: Z_{i}$ is part of the antecedent of a simple minimal theory of $Z_{i+1}$.

\section{The basic idea and input data}

Minimal theories represent deterministic causal structures in a transparent way. Conjunctions in the antecedent of a minimal theory stand for complex causes of the factor in the consequent, disjunctions for alternative causes. Hence, minimal theories are directly causally interpretable. Moreover, minimal theories impose constraints on the behavior of the factors contained in them. For instance, (1) says that whenever $A C$ is instantiated, there also is an instance of $B$. That means, according to (1), the coincidence $A C \bar{B}$ does not occur. Consequently, information about occurring and nonoccurring coincidences allows for conclusions as to the minimal theory representing the underlying causal structure. If it is discovered, say, in a given experimental setup, that $A C$ is never realized in combination with $\bar{B}$, while both $A \overline{C B}$ and $\bar{A} C \bar{B}$ are found to be empirically realized, it follows that $A C$ is minimally sufficient for $B$ relative to the causal background of the corresponding setup. In this sense, minimal theories constitute the link between the empirical behavior of the factors in an investigated 
frame and the deterministic structure behind that behavior. The empirical behavior of the factors allows for inferring minimal theories that describe that behavior, and these theories, in turn, are causally interpretable.

The procedure of causal reasoning to be developed here operates on pure coincidence data with respect to the factors involved in a causal process whose structure is to be revealed-hence the label coincidence analysis. Based on its input data, CNA determines for each factor $Z_{i}$ in the analyzed frame involving, say, $n$ factors which deterministic dependencies hold between $Z_{i}$ and the other $n-1$ factors in the frame. Most of these dependencies will turn out not to be causally interpretable. The possibly causally interpretable dependencies are subsequently minimalized and expressed in terms of minimal theories, which, finally, are straightforwardly causally interpretable as shown above.

The data processed by CNA is listed analogously to truth tables. Tables as in Table 1 are referred to as coincidence lists. The rows in a coincidence list shall be numbered starting with the first row below the title row. The row constituted by " 111 " in list (a) is row 1 (R1), the row featuring "1 01 " is row 2 (R2), and so on. In coincidence lists a ' 1 ' in the column of, say, factor $A$ represents an instance of $A$, a ' 0 ' in that same column symbolizes the absence of such an instance. Columns of coincidence lists thus record instances and absences of the factor mentioned in the title row, while the rows following the title row specify coincidences of the factors in the title row. For example, the first row, $\mathrm{R} 1$, of (a) records the coincidence $A B C$, the following row, $\mathrm{R} 2$, indicates the coincidence $A \bar{B} C$.

List (a) in Table 1 clearly exhibits dependencies among its factors. For instance, there is no row in (a) featuring $A B \bar{C}$. That means the coincidence $A B$ is sufficient for $C$. Likewise, there is no row in (a) featuring $A$ in combination with $\bar{C}$, which amounts to the sufficiency of $A$ for $C$. The sufficient condition $A B$, hence, contains a sufficient proper part, $A$, and, accordingly, is not minimally sufficient. Factor $A$, on the other hand, does not have any sufficient proper parts and, thus, is minimally sufficient for $C$. Analogously it can be shown that $\bar{B} C$ is minimally sufficient for $A$ in list (a). As will be shown below, some of these dependencies are causally interpretable, others are not.

In contrast, list (b) contains all eight logically possible configurations of the three factors in its frame. (b) is therefore referred to as a complete coincidence list. Complete lists do not feature dependencies among their factors. Accordingly, complete lists do not need to be analyzed for dependencies to begin with. Dependencies only emerge in

Table 1 Simple examples of coincidence lists as processed by CNA

\begin{tabular}{|c|c|c|c|c|c|}
\hline & & & $A$ & $B$ & $C$ \\
\hline$A$ & $B$ & $C$ & 1 & 1 & 1 \\
\hline & & & 1 & 1 & 0 \\
\hline 1 & 1 & 1 & 0 & 1 & 1 \\
\hline 1 & 0 & 1 & 1 & 0 & 1 \\
\hline 0 & 1 & 1 & 1 & 0 & 0 \\
\hline 0 & 0 & 0 & 0 & 1 & 0 \\
\hline & (a) & & 0 & 0 & 1 \\
\hline & (a) & & 0 & 0 & 0 \\
\hline
\end{tabular}

(b)

\begin{tabular}{lll}
\hline$A$ & $B$ & $C$ \\
\hline 1 & 1 & 1 \\
0 & 1 & 1 \\
1 & 0 & 1 \\
1 & 0 & 0 \\
0 & 1 & 0 \\
0 & 0 & 1 \\
0 & 0 & 0 \\
\hline
\end{tabular}

(c)

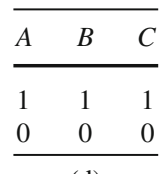

(d) 
incomplete lists, i.e. in lists that feature less than $2^{n}$ coincidences of the $n$ factors in their frame. Upon investigating processes with hard to control causal backgrounds, however, all logically possible factor combinations are no rare empirical result in scientific practice. In such cases, it is often possible to exclude certain configurations as "don't care" cases based on prior causal knowledge. ${ }^{12}$ Alternatively, significance levels may be introduced that exclude rarely found configurations from consideration. ${ }^{13}$ Thus, there are several methodologies available that reduce complete coincidence lists such as to render them interpretable in terms of being the result of deterministic causal structures.

List (c) in Table 1 is not complete in this sense. There is no row in that list such that $A$ and $B$ are instantiated without an instance of $C . A B$ is minimally sufficient for $C$ relative to list (c). Finally, list (d) is incomplete as well. It is incomplete to such an extent that too many dependencies emerge. According to list (d), every factor is minimally sufficient and necessary for every other factor in the corresponding frame. Given such an abundance of dependencies causes and effects cannot be distinguished. As the previous section has shown, if causal dependencies are to be oriented on the basis of mere coincidence data, the analyzed factor frame must include at least two alternative causes for each effect. As in case of complete lists, prior knowledge may provide a means to causally analyze data featuring this kind of insufficient diversity. It is possible that, based on such knowledge, lists as (d) can be supplemented by additional rows representing coincidences that, notwithstanding the fact that they have not been observed in a given study, are known to be empirically possible. As such data adjustment, however, is not part of mechanically uncovering deterministic structures but a precondition thereof, it shall not be further discussed here.

\section{Empirical exhaustiveness and homogeneity}

Apart from the requirement as to the minimal diversity of analyzed coincidence lists, CNA imposes two important constraints on its input data: (I) unambiguous causal inferences are only possible given that the coincidence data is exhaustive and (II) the causal background of coincidence lists must be homogeneous. Let us take these constraints in turn.

Any procedure of causal reasoning, in some way or another, assumes that its input data is exhaustive. Probabilistic procedures presume the availability of probability distributions over all variables in the model space, or Ragin's $(1987$; 2000) QCAalgorithm relies on the realizability of all $2^{m}$ configurations of $m$ cause variables. Nonetheless, assumptions as regards the exhaustiveness of empirical data are hardly ever made explicit in studies on causal reasoning. ${ }^{14}$ Such an implicit taking for granted of the suitability of input data, however, will not do for the present context. As the previous section has shown, deterministic dependencies among $n$ factors emerge only if not all $2^{n}$ coincidences are contained in an analyzed list. Of course, coincidences may not only be missing from coincidence lists due to causal dependencies among

\footnotetext{
12 Cf. Ragin (1987, pp. 113-118).

13 Cf. Ragin (2000, pp. 109-115).

14 One exception is Ragin $(1987 ; 2000)$. He discusses at length how limited empirical data negatively affects causal reasoning.
} 
respective factors. Exhaustive data collection may fail for a host of different reasons. Financial or technical resources may happen to be limited in experimental sciences or nature may be found not to provide sufficient data in non-experimental disciplines. Inexhaustive data is likely to be one of the main reasons for hampered causal interpretability of that data. Minimal theories are only unambiguously assignable to coincidence lists provided that the latter are assumed to be empirically exhaustive in the following sense:

Principle of empirical exhaustiveness (PEX): The collection of empirical data to be processed by CNA faces no practical limitations whatsoever. All coincidences of the analyzed factors that are compatible with the causal structure regulating the behavior of these factors are in fact observed.

(PEX) guarantees that whenever a coincidence is missing from a CNA-processed list, this is due to underlying causal dependencies. Clearly, (PEX) constitutes a sweeping idealization with respect to data collection. Such an idealization, however, may prove to be useful in many practical contexts. It can be implemented as a gauge by means of which concrete data collections can be measured and thus evaluated. For clearly, if there is reason to believe that a particular study did not collect all the relevant data about an investigated structure and if there is no other source available that supplements missing data, the corresponding structure simply cannot be fully uncovered. Accordingly, while (PEX) is a necessary condition for drawing unambiguous inferences, it is not a necessary condition for drawing (ambiguous) causal inferences from coincidence lists. If inexhaustive lists are processed by CNA, as will be shown in Sect. 10 below, more than one minimal theory will be assigned to such lists. The number of minimal theories assigned to an inexhaustive list depends on the logical possibilities of complementing a respective inexhaustive list in a causally interpretable manner. Thus, while it is impossible to infer a single causal structure from an inexhaustive coincidence list, a set of structures can be inferred such that all of its members are compatible with the coincidences recorded in the inexhaustive list. Assigning sets of causal structures to inexhaustive lists, of course, also is a form of causal inference. Such inferences might prove to be of great practical use, for they at least shed light on what structures cannot underly an investigated factor frame. Therefore, CNA does not necessarily have to be based on (PEX). Nonetheless, as failures of (PEX) are a problem of proper data collection and as the latter is not part of causal reasoning per se, but a precondition thereof, (PEX) shall be endorsed in Sects.5-9 which are concerned with matters of causal reasoning only. A detailed discussion of violations of (PEX) is postponed until Sect. 10 .

While violations of (PEX) induce ambiguities in the output of CNA, yet do not give rise to fallacious causal inferences, causal fallacies may result if the causal background of an analyzed coincidence list is not causally homogeneous. A list as (a) in Table 1 could be generated by suitable separate manipulation of each factor. A causal interpretation of such an 'artificial' list, of course, would be fallacious. Causal relevancies would be attributed to factors in the frame which, in fact, did not contribute to the behavior of respective effect factors. Such as to forestall causal fallacies, it must be presumed that the behavior of the factors in the investigated frame is not confounded by causally relevant factors not contained in the frame. Each analysis of 
a causal structure is limited to a small subset of all factors involved in that structure. Causal structures are extremely complex. Ordinarily, only a few factors are of interest in the course of concrete studies. While it is not necessary to assume that a CNA-analyzed coincidence list contains all causally relevant factors involved in an investigated structure, it must be presupposed that a CNA-analyzed coincidence list over a frame consisting of $Z_{1}, \ldots, Z_{n}$ is generated against a causal background that is homogeneous with respect to confounders not contained in $\left\{Z_{1}, \ldots, Z_{n}\right\}$. In order to spell out the notion of a confounder needed for our purposes, the notion of a causal path is required: A sequence of factors $\left\langle Z_{1}, \ldots, Z_{k}\right\rangle, k \geq 2$, constitutes a causal path from $Z_{1}$ to $Z_{k}$ iff for each $Z_{i}$ and $Z_{i+1}, 1 \leq i<k$, in the sequence: $Z_{i}$ is directly causally relevant to $Z_{i+1}$. A condition $X_{j}$ is said to be part of a causal path, if at least one conjunct of $X_{j}$ is contained in the sequence constituting that path. Now the notion of a confounder can be clarified: If $Z_{n}$ is an effect, a confounder of $Z_{n}$ is a minimally sufficient condition $X_{j}$ of $Z_{n}$ such that $X_{j}$ is causally relevant to $Z_{n}$ and $X_{j}$ is part of a causal path leading to $Z_{n}$ not containing any of the factors $Z_{1}, \ldots, Z_{n-1}$. That means a factor $Z_{o}$ that is causally relevant to an effect $Z_{n}$ and that is not contained in the investigated frame $\left\{Z_{1}, \ldots, Z_{n}\right\}$ cannot confound causal reasoning if all causal paths connecting $Z_{o}$ and $Z_{n}$ contain at least one factor in $\left\{Z_{1}, \ldots, Z_{n}\right\}$, i.e. if $Z_{o}$ is a cause or an effect of a factor in the investigated frame. A confounder is a factor or a conjunction of factors by means of which the investigated effect can be manipulated independently of the factors in the frame.

The notion of a confounder is to be understood relative to a corresponding effect. Basically, any factor in an analyzed frame can be seen as an effect of an underlying structure. However, as will be shown below, there are several constraints subject to which a factor can be excluded from the set $\mathrm{W}$ of potential effects contained within a given factor frame prior to causally analyzing that frame. Still, depending on the specific $Z_{i} \in \mathrm{W}$ analyzed in the course of a particular run of CNA, different factors are to be seen as confounders and, accordingly, must be homogenized. Generally: Input data processed by CNA is assumed to be generated against causally homogeneous backgrounds in the sense of (HC):

Homogeneity (HC): The background of a causally analyzed list of $m$ coincidences over a factor frame containing the set $\mathrm{W}$ of potential effects is causally homogeneous iff for every confounder $X_{j}$ of every factor in $\mathrm{W}: X_{j}$ is absent in the background of one coincidence iff $X_{j}$ is absent in the backgrounds of all other $m-1$ coincidences.

While only homogeneous coincidence lists are causally analyzable, (HC) does not guarantee the causal analyzability of coincidence lists. Rather, (HC) prevents causal fallacies. Therefore, a coincidence list may well be homogeneous in terms of (HC), even though confounders are instantiated in its background-as long as these confounders are instantiated in the backgrounds of all coincidences. If confounders are universally instantiated, effects will be present in all coincidences, irrespective of whether the other factors in the frame are present or absent. In this case no dependencies emerge and thus no inferences as to underlying causal structures are drawn. As a consequence, no causal fallacies are committed either. 
Table 2 Two coincidence lists that cannot be causally analyzed, for none of the involved factors can be interpreted as an effect of an underlying causal structure in accordance with (HC)

\begin{tabular}{lll}
\hline$A$ & $B$ & $C$ \\
\hline 1 & 1 & 1 \\
1 & 0 & 1 \\
0 & 1 & 1 \\
1 & 1 & 0 \\
\hline
\end{tabular}

(a)

\begin{tabular}{lll}
\hline$A$ & $B$ & $C$ \\
\hline 1 & 0 & 0 \\
0 & 1 & 0 \\
0 & 0 & 1 \\
0 & 0 & 0 \\
\hline
\end{tabular}

(b)

(HC) excludes a number of coincidence lists from causal analyzability. The lists fed into CNA may well reveal certain backgrounds to be causally inhomogeneous. Consider, for instance, the lists in Table 2. Assume $B$ to be an effect of the causal structure generating list (a) in Table 2. A comparison of the coincidences recorded in rows 1 (R1) and 2 (R2) shows that, if $B$ in fact were the effect of the underlying structure, list (a) would violate (HC). The only factor varying in R1 and R2 is $B$; no other factor in the frame $\{A, B, C\}$ is accountable for that variation of $B$, therefore, it must be due to a varying confounder of $B$ in the unknown or unconsidered background of list (a). That means assuming $B$ to be an effect contradicts the homogeneity assumption. In contrast, if $B$ is taken to be a cause factor of the underlying structure, (HC) is not violated. Thus, assuming (HC) to hold for list (a) implies that $B$ cannot be seen as a possible effect. The same holds for the other two factors in $\{A, B, C\}$. In R1 and R3 $A$ is the only varying factor, while no other factor, apart from $C$, varies in R1 and R4. Hence, there is no factor in list (a) that could possibly be an effect of an underlying causal structure in accordance with (HC). Analogous considerations apply to list (b) of Table 2 .

That means there cannot be a causal structure underlying either list (a) or (b) that would be compatible with (HC). Neither list comprises a factor that could be seen as an effect in accordance with (HC), i.e. $W=\emptyset$. Whenever for every factor $Z_{i}$ contained in the factor frame of a coincidence list $\mathcal{C}$ there are two rows $\mathrm{R} k$ and $\mathrm{R} l$ in $\mathcal{C}$ such that $Z_{i}$ is the only factor varying in $\mathrm{R} k$ and $\mathrm{R} l$, the background against which the data in $\mathcal{C}$ is collected cannot be homogeneous, for there is no causal structure that could possibly generate $\mathcal{C}$ and accord with (HC). I shall in this context speak of inhomogeneous coincidence lists. (HC) excludes all inhomogeneous coincidence lists from being processed by CNA. It must be emphasized, however, that the homogeneity of coincidence lists is an assumption to which every inference of CNA must be relativized. It might well be that a list which is not inhomogeneous in the sense defined above, as e.g. list (a) in Table 1, in fact is the result of an uncontrolled variation of background confounders. In this sense, only a sufficient and no necessary condition for the inhomogeneity of a coincidence list is given above. Causal inferences drawn by CNA will always be of the form "Given that (HC) is satisfied, such and such are the underlying causal structure(s)". Homogeneity is never beyond doubt.

Generally, determining whether empirical exhaustiveness and homogeneity are satisfied ultimately calls for some form of inductive justification which, however, is not going to be discussed in the present context. Empirical exhaustiveness and homogeneity shall simply be taken to bear the inductive risk that comes with drawing causal inferences based on CNA. 


\section{Identification of potential effects}

After having clarified the presuppositions on which CNA rests, we can now proceed to introduce the inference rules of CNA. As anticipated in the previous section, a first algorithmic step consists in parsing through the factor frame of a coincidence list in order to determine which of the factors could possibly operate as effects within the causal structure to be revealed. This step yields a set $\mathrm{W}$ of factors whose dependencies on the other factors in the frame are then successively determined by CNA. The identification of potential effects shall not be considered a proper part of CNA, for any sort of context-dependent empirical information or even prior causal knowledge is allowed to enter the determination of W. For instance, if, in a given experimental setup, a factor $Z_{i}$ is generally instantiated temporally before every other factor in an analyzed frame $\left\{Z_{1}, \ldots, Z_{n}\right\}, Z_{i}$ cannot function as an effect within the underlying structure. Or prior causal knowledge could be available that establishes the members of a proper subset of $\left\{Z_{1}, \ldots, Z_{n}\right\}$ as root factors, i.e. as factors that are causes, but no effects within a causal structure. In both cases there is no need to integrate respective factors in W. CNA does not have to evaluate dependencies among factors that can be excluded from the set of potential effects to begin with. These context-dependent constraints on $\mathrm{W}$ are not systematizable or, at least, a systematization shall not be attempted here. Accordingly, no recursively applicable or computable rule can be provided, which essentially is why the determination of $W$ is not seen as a proper part of CNA.

Still, the determination of $\mathrm{W}$ is not only regulated by spatiotemporal peculiarities of an analyzed process or by prior causal knowledge. As the previous section has shown, factors can be excluded from the set of potential effects based on homogeneity considerations: In order for a factor $Z_{i}$ to be a potential effect, it must not be the case that the corresponding coincidence list contains two rows such that $Z_{i}$ is the only varying factor in those rows. Furthermore, since CNA shall be designed to infer causes of both positive and negative factors, $\mathrm{W}$, in principle, may contain both positive and negative factors. However, to every minimal theory of a positive factor $Z_{i}$, there exists an equivalent minimal theory of $\overline{Z_{i}}$, and vice versa.

(5) $A C \vee D E \Rightarrow B$

(6) $\overline{A D} \vee \overline{A E} \vee \overline{C D} \vee \overline{C E} \Rightarrow \bar{B}$

(5) and (6) are logically equivalent and one of these expressions is a minimal theory if and only if the other one is too. ${ }^{15}$ Hence, for simplicity's sake, CNA can be confined to identify minimal theories of either positive factors or their negative counterparts. For this reason, we stipulate that positive factors only shall be included in W.

These considerations taken together yield the following standard as regards the determination of $\mathrm{W}$. In order to indicate that the non-computable identification of the set of potential effects is a precondition of launching CNA, yet not a proper part thereof, it shall be referred to as "step $0 *$ ".

\footnotetext{
15 For a detailed proof of the existence of an equivalent minimal theory of a negative factor to every minimal theory of a positive factor cf. Baumgartner (2006, Chap. 3).
} 
Step $0 *$ - Identification of potential effects: Given a coincidence list $\mathcal{C}$ over a factor frame $\left\{Z_{1}, \ldots, Z_{n}\right\}$, identify the subset $\mathrm{W} \subseteq\left\{Z_{1}, \ldots, Z_{n}\right\}$ such that for every $Z_{i}: Z_{i} \in \mathrm{W}$ iff

(1) The totality of available information as to the spatiotemporal ordering of the instances of the factors in $\left\{Z_{1}, \ldots, Z_{n}\right\}$ and the available prior causal knowledge about the behavior of the factors in $\left\{Z_{1}, \ldots, Z_{n}\right\}$ does not preclude $Z_{i}$ to be an effect of the underlying causal structure.

(2) $\mathcal{C}$ does not contain two rows $\mathrm{R} k$ and $\mathrm{R} l$ such that $Z_{i}$ is the only factor varying in the coincidences recorded by $\mathrm{R} k$ and $\mathrm{R} l$.

(3) $Z_{i}$ is a positive factor.

\section{Identification and minimalization of sufficient conditions}

After having identified a non-empty set of potential effects, CNA proper sets in. In a first stage, sufficient conditions for each member of $W$ are identified and minimalized. In order to illustrate this first stage, let us look at a concrete example. Assume the coincidence list depicted in Table 3 to be our input data. None of the factors in our exemplary frame $\{A, B, C, D, E\}$ shall be excluded from effect position by prior causal knowledge or additional information as to spatiotemporal orderings. Nonetheless, the set of potential effects does not correspond to the factor frame of Table 3, i.e. $\mathrm{W} \neq\{A, B, C, D, E\}$. For reasons of compatibility with (HC), $A, B$, and $D$ cannot be effects. For each of these factors there is a pair of rows in Table $3-\langle\mathrm{R} 1, \mathrm{R} 4\rangle$ for $A,\langle\mathrm{R} 1, \mathrm{R} 3\rangle$ for $B,\langle\mathrm{R} 1, \mathrm{R} 2\rangle$ for $D$-such that the respective factor is the only varying factor. Thus, interpreting one of these factors to be an effect of the underlying structure would contradict CNA's homogeneity assumption. $C$ and $E$, thus, are the only potential effects of the structure generating Table 3, i.e. $\mathrm{W}=\{C, E\}$. For each of the factors in $W$ minimally sufficient conditions are now identified. This is done in four steps: (1) a factor $Z_{i} \in \mathrm{W}$ is selected, (2) sufficient conditions of $Z_{i}$ are identified, (3) these sufficient conditions are minimalized, (4) the procedure is restarted at (1) by selecting another $Z_{j} \in \mathrm{W}$, until all factors in $\mathrm{W}$ have been selected. Let us take a detailed look at these four steps.

Step 1-Selection of a potential effect: Randomly select one factor $Z_{i} \in$ W such that $Z_{i}$ has not been selected in a previous run of steps $1-4$. $Z_{i}$ is termed effect $t^{*}$, the factors in $\left\{Z_{1}, \ldots, Z_{i-1}, Z_{i+1}, \ldots, Z_{n}\right\}$ are referred to as remainders. ${ }^{16}$

Step 2-Identification of sufficient conditions: Identify all sufficient conditions of the effect* $Z_{i}$ according to the following rule:

\footnotetext{
16 Selected factors are labeled effects* to indicate that they possibly are the effects of the causal structure generating the input list. Effects* do not necessarily turn out to be (actual) effects at the end of a CNA-analysis. For instance, the set of effects* contained in list (d) of Table 1 contains all factors in the frame-provided no further information is available that distinguishes among causes and effects. Yet, none of these effects* is identified as an actual effect by CNA, because causes and effects cannot be kept apart relative to that list.
} 
Table 3 Exemplary

coincidence list to be analyzed by CNA

\begin{tabular}{lllll}
\hline$A$ & $B$ & $C$ & $D$ & $E$ \\
\hline 1 & 1 & 1 & 1 & 1 \\
1 & 1 & 1 & 0 & 1 \\
1 & 0 & 1 & 1 & 1 \\
0 & 1 & 1 & 1 & 1 \\
0 & 1 & 1 & 0 & 1 \\
1 & 0 & 1 & 0 & 0 \\
0 & 0 & 0 & 1 & 1 \\
0 & 0 & 0 & 0 & 0 \\
\hline
\end{tabular}

(SUF) A coincidence $X_{k}$ of remainders is sufficient for $Z_{i}$ iff the input list $\mathcal{C}$ contains at least one row featuring $X_{k} Z_{i}$ and no row featuring $X_{k} \overline{Z_{i}}$.

The order of selecting effects* in step 1 does not matter, as long as it is guaranteed that, eventually, all members of W are selected. According to (SUF), a coincidence of remainders can only be sufficient for an effect* if it is instantiated at least once. Moreover, a coincidence of remainders contained in the input list is not sufficient for a selected effect* if it is also instantiated in combination with the absence of that effect*.

Let us perform these two steps on our example of Table 3 by first selecting $C$ as effect*. Step 2 identifies six sufficient conditions of $C$, i.e. there are six coincidences of remainders that conform to (SUF): $A B D E, A B \bar{D} E, A \bar{B} D E, \bar{A} B D E, \bar{A} B \bar{D} E$, $A \overline{B D E}$. The first row (R1) of Table 3 features the coincidence $A B D E$ in combination with $C$ and there is no row such that $A B D E$ is contained therein in combination with $\bar{C} . A B D E$, thus, is a sufficient condition of $C$ according to (SUF). Analogous considerations apply to the other sufficient conditions mentioned above: R2 is constituted by $A B \bar{D} E$, R3 by $A \bar{B} D E$, R4 by $\bar{A} B D E$, R5 by $\bar{A} B \bar{D} E$, and R6 features $A \overline{B D E}$ without either of these conditions being contained in combination with $\bar{C}$ in Table 3 .

Before sufficient conditions of the remaining effect* $E$ are identified, we proceed to minimalize the sufficient conditions of $C$.

Step 3-Minimalization of sufficient conditions: The sufficient conditions of $Z_{i}$ identified in step 2 are minimalized according to the following rule:

(MSUF) A sufficient condition $Z_{1} Z_{2} \ldots Z_{h}$ of $Z_{i}$ is minimally sufficient iff neither $Z_{2} Z_{3} \ldots Z_{h}$ nor $Z_{1} Z_{3} \ldots Z_{h}$ nor $\ldots$ nor $Z_{1} Z_{2} \ldots Z_{h-1}$ are sufficient for $Z_{i}$ according to (SUF).

Or operationally put:

$\left(\right.$ MSUF $\left.^{\prime}\right)$ Given a sufficient condition $Z_{1} Z_{2} \ldots Z_{h}$ of $Z_{i}$, for every $Z_{g}$ $\in\left\{Z_{1}, Z_{2}, \ldots, Z_{h}\right\}, h \geq g \geq 1$, and every $h$-tuple $\left\langle Z_{1^{\prime}}, Z_{2^{\prime}}, \ldots, Z_{h^{\prime}}\right\rangle$ which is a permutation of the $h$-tuple $\left\langle Z_{1}, Z_{2}, \ldots, Z_{h}\right\rangle$ : Eliminate $Z_{g}$ from $Z_{1} Z_{2} \ldots Z_{h}$ and check whether $Z_{1} \ldots Z_{g-1} Z_{g+1} \ldots Z_{h} \overline{Z_{i}}$ is contained in a row of $\mathcal{C}$. If that is the case, re-add $Z_{g}$ to $Z_{1} \ldots Z_{g-1} Z_{g+1} \ldots$ $Z_{h}$ and eliminate $Z_{g+1}$; if that is not the case, proceed to eliminate $Z_{g+1}$ without re-adding $Z_{g}$. The result of performing this redundancy check on every factor contained in $Z_{1} Z_{2} \ldots Z_{h}$ is a set of minimally sufficient conditions of $Z_{i}$. 
(MSUF) is nothing but an adaptation of the notion of a minimally sufficient condition as defined in Sect. 2 to the context of coincidence lists. $\left(M_{S U F}^{\prime}\right)$, on the other hand, can be seen as an operational expression of the analysans of the notion of a minimally sufficient condition implemented in (MSUF). That is, (MSUF) might be rephrased as follows: A sufficient condition $Z_{1} Z_{2} \ldots Z_{h}$ of $Z_{i}$ is minimally sufficient iff it results from an application of $\left(\mathrm{MSUF}^{\prime}\right)$. At the expense of high computational complexity, the formulation of $\left(\mathrm{MSUF}^{\prime}\right)$ is kept as simple as possible above. The order in which factors are eliminated from sufficient conditions matters as to the minimalization of such conditions - thus the systematic permutation of elimination orders. ${ }^{17}$ In many cases, however, it is not necessary to completely perform that permutation. For instance, assume an $h$-tuple $T_{1}=\left\langle Z_{1}, \ldots, Z_{d}, Z_{d+1}, \ldots, Z_{h}\right\rangle$ has been minimalized by means of $\left(\mathrm{MSUF}^{\prime}\right)$ up to element $Z_{d}$, that minimalization of $T_{1}$ can be taken over for all $h$-tuples $T_{2}=\left\langle Z_{1}, \ldots, Z_{d}, Z_{d+1^{\prime}}, \ldots, Z_{h^{\prime}}\right\rangle$ that coincide with $T_{1}$ up to element $Z_{d}$ without reapplying $\left(\mathrm{MSUF}^{\prime}\right)$ to $T_{2}$. Or suppose it has been found that $X_{1}=Z_{1} \ldots Z_{d}$ is a minimally sufficient condition of an investigated effect and a sufficient condition $X_{2}=Z_{1} Z_{2} \ldots Z_{h}$ containing $Z_{1} \ldots Z_{d}$ is to be minimalized by means of $\left(\mathrm{MSUF}^{\prime}\right)$. In that case, it is not effective to minimalize $X_{2}$ by first eliminating the factors not contained in $X_{1}$, for this elimination order would just yield $X_{1}$ again.

Further optimizations of (MSUF') are conceivable, yet are not going to be discussed here - they will have to await another paper. More importantly, the intuition behind $\left(\mathrm{MSUF}^{\prime}\right)$ can be more colloquially captured: Every factor contained in a sufficient condition of $Z_{i}$ is to be tested for redundancy by eliminating it from that condition and checking whether the remaining condition still is sufficient for $Z_{i}$ or not. A sufficient condition of $Z_{i}$ is minimally sufficient iff every elimination of a factor from that condition results in the insufficiency of the remaining condition.

Performing step 3 on our exemplary case is straightforward. Step 2 yielded six sufficient conditions of $C$. For brevity, I only illustrate the minimalization of these six conditions by means of two examples. First, take $A B D E$. That this sufficient condition is not minimally sufficient for $C$ is seen by removing, say, $D$ and finding that $A B E$ itself is sufficient for $C$, for Table 3 does not contain a row featuring $A B E$ in combination with $\bar{C}$. $A B E$ still is not minimally sufficient. For instance, both $B$ and $E$ can be removed without sufficiency being lost. There is no row in Table 3 featuring $A \bar{C}$, which induces that $A$ is sufficient and, since it is a single factor that does not contain proper parts, minimally sufficient for $C$. There are other ways to further minimalize $A B E$ : A removal of $A$ and $E$ still yields a sufficient condition of $C$. There is no row in Table 3 featuring $B \bar{C}$. Therefore $B$ is minimally sufficient for $C$. Second, let us look at the second sufficient condition of $C$ identified by (SUF). $A B \bar{D} E$ is not minimally sufficient because $A B$ can be removed without sufficiency for $C$ being lost. There is no row in Table 3 featuring $\bar{D} E$ in combination with $\bar{C}$, which induces that $\bar{D} E$ is sufficient for $C$. If $\bar{D} E$ is further reduced, sufficiency is lost. R7 features

\footnotetext{
17 This is an important deviation from the minimalization of sufficient conditions in the vein of the QuineMcCluskey optimization of truth functions. Quine-McCluskey optimization only eliminates conjuncts of a sufficient condition if the latter reduced by the respective conjunct is actually contained in the truth table. As will become apparent in Sect. 9, this restriction is a serious limitation of the minimizability of sufficient conditions involved in chainlike causal structures.
} 
$\bar{C} E$ and R8 $\overline{C D} \cdot \bar{D} E$, hence, is minimally sufficient for $C$. Minimalizing the other sufficient conditions of $C$ by analogously implementing $\left(\mathrm{MSUF}^{\prime}\right)$ does not yield any further minimally sufficient conditions. All in all, therefore, minimalizing the sufficient conditions of $C$ generates the following three minimally sufficient conditions: $A, B$, and $\bar{D} E$.

After having identified the minimally sufficient conditions of a first factor $Z_{i} \in \mathrm{W}$, the same needs to be done for all other effects*. We thus need a loop that brings CNA back to step 1 , if not all factors in W have been assigned minimally sufficient conditions yet.

Step 4-(MSUF)-Loop: If all $Z_{i} \in \mathrm{W}$ have been selected as effects* proceed to step 5, otherwise go back to step 1 .

Applying this loop to our example yields six sufficient conditions of $E$. Each row featuring $E$ comprises a sufficient condition of remainders: $A B C D, A B C \bar{D}, A \bar{B} C D$, $\bar{A} B C D, \bar{A} B C \bar{D}, \overline{A B C} D$. For example, $\mathrm{R} 2$ of Table 3 is constituted by $A B C \bar{D}$ and there is no row featuring $A B C \bar{D}$ along with $\bar{E}$, or R3 comprises $A \bar{B} C D$ and no row in Table 3 contains $A \bar{B} C D$ in combination with $\bar{E}$. The sufficiency of the other conditions is analogously demonstrated. Employing (MSUF) or $\left(\mathrm{MSUF}^{\prime}\right)$ to minimalize these conditions brings forth three minimally sufficient conditions of $E$ : $B, D$, and $\bar{A} C$. The list in Table 3 contains no rows featuring either $B \bar{E}, D \bar{E}$, or $\bar{A} C \bar{E}$.

As an overall result of performing the first stage (steps 1 to 4 ) of CNA on our exemplary case, we have thus identified the following minimally sufficient conditions of the factors in W:
$A, B, \bar{D} E$ for $C$,
$B, D, \bar{A} C$ for $E$.

\section{Identification and minimalization of necessary conditions}

As the famous Manchester Hooters counterexample against Mackie's (1974) INUS-theory of causation ${ }^{18}$ demonstrates and as articulated in the analysis of causal relevance given in Sect. 2, minimally sufficient conditions are not generally causally interpretable. Only minimally sufficient conditions that are moreover non-redundant parts of minimally necessary conditions are amenable to a causal interpretation. After having identified minimally sufficient conditions, we thus now proceed to first form necessary conditions of the effects* from their minimally sufficient conditions and then minimalize these necessary conditions. Since factor frames processed by CNA are incomplete with respect to underlying causal structures, i.e. there supposedly will always be many causally relevant factors not contained in input lists, effects* can only be assigned necessary conditions relative to the homogeneous backgrounds of corresponding coincidence lists. This is easily accomplished by disjunctively combining the minimally sufficient conditions of each effect*, yielding one necessary condition relative to an input list $\mathcal{C}$ and its background for each factor $Z_{i} \in \mathrm{W}$.

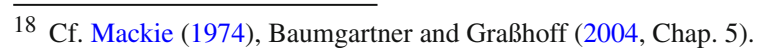


Step 5-Identification of necessary conditions: Identify a necessary condition of each effect* $Z_{i}$ by disjunctively concatenating $Z_{i}$ 's minimally sufficient conditions according to the following rule:

(NEC) A disjunction $X_{1} \vee X_{2} \vee \cdots \vee X_{h}$ of minimally sufficient conditions of $Z_{i}$ is necessary for $Z_{i}$ iff $\mathcal{C}$ contains no row featuring $Z_{i}$ in combination with $\neg\left(X_{1} \vee X_{2} \vee \cdots \vee X_{h}\right)$, i.e. no row comprising $\overline{X_{1} X_{2} \ldots X_{h}} Z_{i}$.

Performed on our example, step 5 issues $A \vee B \vee \bar{D} E$ and $B \vee D \vee \bar{A} C$ as necessary conditions of $C$ and $E$, respectively. Such as to determine whether the minimally sufficient conditions assigned to the effects* at the end of the previous section in fact are non-redundant parts of necessary conditions, these necessary conditions have to be minimalized.

Step 6-Minimalization of necessary conditions: The necessary conditions of every $Z_{i} \in \mathrm{W}$ identified in step 5 are minimalized according to the following rule:

(MNEC) A necessary condition $X_{1} \vee X_{2} \vee \cdots \vee X_{h}$ of $Z_{i}$ is minimally necessary iff neither $X_{2} \vee X_{3} \vee \cdots X_{h}$ nor $X_{1} \vee X_{3} \vee \cdots X_{h}$ nor ... nor $X_{1} \vee X_{2} \vee \cdots \vee X_{h-1}$ are necessary for $Z_{i}$ according to (NEC).

Or operationally put:

$\left(\mathrm{MNEC}^{\prime}\right)$ Given a necessary condition $X_{1} \vee X_{2} \vee \cdots \vee X_{h}$ of $Z_{i}$, for every $X_{g} \in\left\{X_{1}, X_{2}, \ldots, X_{h}\right\}, h \geq g \geq 1$, and every $h$-tuple $\left\langle X_{1^{\prime}}, X_{2^{\prime}}, \ldots\right.$, $\left.X_{h^{\prime}}\right\rangle$ which is a permutation of the $h$-tuple $\left\langle X_{1}, X_{2}, \ldots, X_{h}\right\rangle$ : Eliminate $X_{g}$ from $X_{1} \vee X_{2} \vee \cdots \vee X_{h}$ and check whether there is a row in $\mathcal{C}$ featuring $Z_{i}$ in combination with $\neg\left(X_{1} \vee \cdots \vee X_{g-1} \vee X_{g+1} \vee \cdots \vee X_{h}\right)$, i.e. a row comprising $\overline{X_{1} \ldots X_{g-1} X_{g+1} \ldots X_{h}} Z_{i}$. If that is the case, re-add $X_{g}$ to $X_{1} \vee \cdots \vee X_{g-1} \vee X_{g+1} \vee \cdots \vee X_{h}$ and eliminate $X_{g+1}$; if that is not the case, proceed to eliminate $X_{g+1}$ without re-adding $X_{g}$. The result of performing this redundancy check on every minimally sufficient condition contained in $X_{1} \vee X_{2} \vee \cdots \vee X_{h}$ is a set of minimally necessary conditions of $Z_{i}$.

In analogy to (MSUF), (MNEC) is nothing but an adaptation of the notion of a minimally necessary condition as defined in Sect. 2 to the context of coincidence lists. $\left(\mathrm{MNEC}^{\prime}\right)$, on the other hand, can be seen as an operational expression of the analysans of the notion of a minimally necessary condition implemented in (MNEC). That means (MNEC) might be rephrased as follows: A necessary condition $X_{1} \vee X_{2} \vee \cdots \vee X_{h}$ is minimally necessary iff it results from an application of $\left(\mathrm{MNEC}^{\prime}\right)$. The formulation of $\left(\mathrm{MNEC}^{\prime}\right)$ has been kept as simple as possible at the expense of its computational complexity. Analogous optimizations as in case of $\left(\mathrm{MSUF}^{\prime}\right)$ are possible with respect to $\left(\mathrm{MNEC}^{\prime}\right)$. The intuition behind $\left(\mathrm{MNEC}^{\prime}\right)$ can also be more colloquially captured: Every minimally sufficient condition contained in a necessary condition of $Z_{i}$ is to be tested for redundancy by eliminating it from that condition and checking whether the remaining condition still is necessary for $Z_{i}$ or not. A necessary condition of $Z_{i}$ 
is minimally necessary iff every elimination of a minimally sufficient condition from that necessary condition results in the loss of necessity of the remaining condition.

Let us illustrate the minimalization of necessary conditions by first performing step 6 on the necessary condition $A \vee B \vee \bar{D} E$ of $C$. That disjunction is not minimally necessary for $C$, because it contains a necessary proper part: $A \vee B$. Whenever $C$ is instantiated in Table 3 , there is an instance of either $A$ or $B$. Table 3 does not contain a row featuring $\overline{A B} C . \bar{D} E$ does not amount to a non-redundant part of a minimally necessary condition, for whenever $\bar{D} E$ is instantiated in combination with $C$, there also is an instance of $A \vee B$. The same results from applying $\left(\mathrm{MNEC}^{\prime}\right)$ to $A \vee B \vee \bar{D} E$. When eliminating $A$ we find that the rest is no longer necessary for $C$, because R3 of Table 3 features $\overline{B \bar{D}} E$ and $C$ or, more specifically, $\bar{B} D E$ and $C$. Hence, $A$ is re-added. The same is found upon removing $B$. R5 features $\overline{A \bar{D}} E$ and $C$ or $\bar{A} D E$ and $C$, respectively. Removing $\bar{D} E$, however, does not result in a loss of necessity. Therefore, $\bar{D} E$ is not re-added. For analogous reasons, $B \vee D \vee \bar{A} C$ does not amount to a minimally necessary condition of $E$ either. $B \vee D \vee \bar{A} C$ contains a necessary proper part: $B \vee D$. There is no row in Table 3 featuring $\overline{B D} E . \bar{A} C$ is not part of a minimally necessary condition of $E$, for whenever $\bar{A} C$ is instantiated, so is $B \vee D$, but not vice versa. All in all, therefore, we get the following minimally necessary conditions for our example:

$$
\begin{array}{ll}
A \vee B & \text { for } C, \\
B \vee D & \text { for } E .
\end{array}
$$

\section{Framing minimal theories and causal interpretation}

In the remaining step of CNA minimal theories are framed from the minimally necessary disjunctions of minimally sufficient conditions identified for each $Z_{i} \in \mathrm{W}$ in step 6. This is done by means of a twofold procedure: First, simple minimal theories are formed for each $Z_{i} \in \mathrm{W}$, and second, if the minimal theories $\Phi$ and $\Psi$ of two different factors in $W$ have a non-empty intersection of factors, $\Phi$ and $\Psi$ are combined to form the complex minimal theory $\Phi \wedge \Psi$, such that $\Phi \wedge \Psi$ conforms to the requirements imposed on the notion of a complex minimal theory in Sect. 2.

Step 7-Framing minimal theories: The minimally necessary disjunctions of minimally sufficient conditions of each $Z_{i} \in \mathrm{W}$ identified in step 6 are assembled to minimal theories as follows:

(1) For each $Z_{i} \in \mathrm{W}$ and each minimally necessary disjunction $X_{1} \vee X_{2} \vee$ $\cdots \vee X_{h}, h \geq 2,{ }^{19}$ of minimally sufficient conditions of $Z_{i}$ : Form a simple minimal theory $\Psi$ of $Z_{i}$ by making $X_{1} \vee X_{2} \vee \cdots \vee X_{h}$ the antecedent of a double-conditional and $Z_{i}$ its consequent: $X_{1} \vee X_{2} \vee \cdots \vee X_{h} \Rightarrow Z_{i}$.

(2) Conjunctively combine two simple minimal theories $\Phi$ and $\Psi$ to the complex minimal theory $\Phi \wedge \Psi$ iff $\Phi$ and $\Psi$ conform to the following conditions:

\footnotetext{
19 The constraint as to a minimum of two alternative minimally sufficient conditions for each effect* does justice to the minimal complexity of a causal structure required such that its direction is identifiable (cf. Sect. 2).
} 
(a) at least one factor in $\Phi$ is part of $\Psi$;

(b) $\Phi$ and $\Psi$ do not have an identical consequent.

In our exemplary case, step 6 of CNA generates one minimally necessary disjunction of minimally sufficient conditions for each $Z_{i} \in \mathrm{W}$. As we shall see in Sect. 9, step 6 sometimes identifies more than one minimally necessary disjunction for certain $Z_{i} \in \mathrm{W}$. Yet, before we look at ambiguities arising when it comes to framing minimal theories let us conclude the analysis of our first exemplary coincidence list. Step 7.1 straightforwardly yields one simple minimal theory for $C$ and $E$ each: $A \vee B \Rightarrow C, B \vee D \Rightarrow E$. While these theories hold for the specific causal background of Table 3, it must not be the case that $A$ and $B$ are themselves sufficient for $C$, or $B$ and $D$ are sufficient for $E$. Moreover, there may well be further minimally sufficient conditions of both $C$ and $E$. Therefore, suspending the relativization to the background of Table 3 and expressing these dependencies in their general and background independent form leads to:

(7) $A X_{1} \vee B X_{2} \vee Y_{C} \Rightarrow C$

(8) $B X_{3} \vee D X_{4} \vee Y_{E} \Rightarrow E$

The simple minimal theories of $C$ and $E$ share one common factor. The causal structure regulating the behavior of $E$ is not independent of the structure behind the behavior of $C$ and vice versa. The behavior of the factors in Table 3, thus, is regulated by a complex structure. Accordingly, step 7.2 of CNA urges us to conjunctively combine (7) and (8) to a complex minimal theory. All in all, step 7 assigns the following complex and background independent minimal theory to the coincidence list in Table 3:

(9) $\left(A X_{1} \vee B X_{2} \vee Y_{C} \Rightarrow C\right) \wedge\left(B X_{3} \vee D X_{4} \vee Y_{E} \Rightarrow E\right)$

After having assigned a minimal theory to a coincidence list, the by far most intricate hurdles on the way to uncovering the deterministic causal structure behind that list have been overcome. As we have seen in Sect. 2, there exists a straightforward syntactical convention as regards the causal interpretation of minimal theories. Minimal theories render causal structures syntactically transparent:

Step 8*-Causal interpretation: Disjuncts in the antecedent of simple minimal theories are to be interpreted as alternative (complex) causes of the factor in the consequent. Conjuncts constituting such disjuncts correspond to non-redundant parts of complex causes. Triples of factors $\left\langle Z_{h}, Z_{i}, Z_{j}\right\rangle$, such that $Z_{h}$ appears in the antecedent of a minimal theory of $Z_{i}$ and $Z_{i}$ is part of a minimal theory of $Z_{j}$, are to be interpreted as causal chains.

This interpretation rule is not to be seen as part of CNA proper. Nonetheless, it fulfills an essential function on the way to a causal inference. For this reason, the rule concerning causal interpretation is starred.

CNA thus determines the coincidences in our exemplary Table 3 to be the result of a deterministic common cause structure: $A$ and $B$ are parts of alternative causes of $C$, while $B$ and $D$ are parts of alternative causes of $E$. Steps $0^{*}$ to 7 assign a minimal theory to a coincidence list and step $8 *$ causally interprets that theory. 


\section{Multiple theories}

As is well known from the literature on causal Bayesian networks, empirical data is not always unambiguously identifiable to be the result of one particular causal structure. At times, data could stem from more than one structure. Such ambiguities are not a particularity of the probabilistic data processed by procedures analyzing causal Bayes nets, for, as the exemplary coincidence list analyzed in this section illustrates, ambiguities can also arise in case of deterministic data. Consider the list in Table 4. It covers the same factor frame as Table 3 and only differs from the latter with respect to one single row: R6. In order to determine the set $\mathrm{W}$ of potential effects, it again is assumed that no factor in $\{A, B, C, D, E\}$ is excluded from effect position by prior causal knowledge or spatiotemporal constraints. For reasons of compatibility with (HC), however, factors $A, B$, and $D$ cannot be effects. Thus, as in case of Table $3, C$ and $E$ are the only potential effects, i.e. $\mathrm{W}=\{C, E\}$.

Performing steps 2 and 3 on $C$ and $E$ yields the following:

Sufficient conditions of $C: A B D E, A B \bar{D} E, A \bar{B} D E, \bar{A} B D E, \bar{A} B \bar{D} E, A \overline{B D} E$.

Minimally sufficient conditions of $C: A, B, \bar{D} E$.

Sufficient conditions of $E$ : $A B C D, A B C \bar{D}, A \bar{B} C D, \bar{A} B C D, \bar{A} B C \bar{D}, A \bar{B} C \bar{D}$,

$\overline{A B C D}$.

Minimally sufficient conditions of $E: A, B, C, D$.

After having identified minimally sufficient conditions, CNA proceeds to first form and then minimalize necessary conditions for each effect*.

Necessary condition of $C: A \vee B \vee \bar{D} E$.

Minimally necessary condition of $C: A \vee B$.

Necessary condition of $E: A \vee B \vee C \vee D$.

Minimally necessary conditions of $E: A \vee B \vee D, C \vee D$.

The necessary condition of $C, A \vee B \vee \bar{D} E$, contains a necessary proper part, viz. $A \vee B$. Whenever $C$ is instantiated in Table 4, there is an instance of either $A$ or $B$. $\bar{D} E$ does not amount to a non-redundant part of a minimally necessary condition, for whenever $\bar{D} E$ is instantiated in combination with $C$, there also is an instance of $A \vee B$. The necessary condition of $E, A \vee B \vee C \vee D$, not only contains one but two necessary proper parts: $C \vee D$ and $A \vee B \vee D$. There is no row in Table 4 featuring $\overline{C D} E$ or $\overline{A B D} E$. Whenever $E$ is instantiated, there is an instance of $C \vee D$ and of $A \vee B \vee D$. These two ways to minimalize $A \vee B \vee C \vee D$ stem from the fact that

Table 4 A second exemplary coincidence list over the same factor frame as the list in Table 3

\begin{tabular}{lllll}
\hline$A$ & $B$ & $C$ & $D$ & $E$ \\
\hline 1 & 1 & 1 & 1 & 1 \\
1 & 1 & 1 & 0 & 1 \\
1 & 0 & 1 & 1 & 1 \\
0 & 1 & 1 & 1 & 1 \\
0 & 1 & 1 & 0 & 1 \\
1 & 0 & 1 & 0 & 1 \\
0 & 0 & 0 & 1 & 1 \\
0 & 0 & 0 & 0 & 0 \\
\hline
\end{tabular}


Fig. 1 A causal chain and a common cause structure that both could underly the coincidences recorded in Table 4

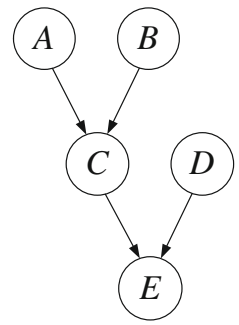

(a)

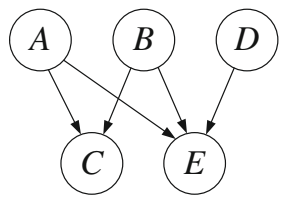

(b)

there are biconditional dependencies among the minimally sufficient conditions of $E$. Within the homogeneous background of Table 4, $C$ is instantiated if and only if $A \vee B$ is instantiated.

Drawing on this inventory of minimally necessary conditions CNA frames one simple minimal theory for $C$ and two for $E$ :

(10) $A X_{1} \vee B X_{2} \vee Y_{C} \Rightarrow C$

(11) $A X_{3} \vee B X_{4} \vee D X_{5} \vee Y_{E} \Rightarrow E$

(12) $C X_{6} \vee D X_{7} \vee Y_{E} \Rightarrow E$

The simple minimal theories of $C$ and $E$ share common factors. The behavior of the factors in Table 4, thus, is regulated by a complex causal structure. In order to determine what that structure looks like, the simple minimal theories of $C$ and $E$ are to be conjunctively combined to form a complex theory. Here an ambiguity emerges: (11) and (12) - if causally interpreted-identify different direct causal relevancies for $E$. While according to (11) $A$ and $B$ are directly causally relevant to $E$, (12) instead holds $C$ to be directly relevant to $E$. The coincidences in Table 4 are either generated by a causal chain such that $A$ and $B$ are parts of alternative causes of $C$ while $C$ and $D$ are contained in alternative causes of $E$, or they are generated by a common cause structure such that $A$ and $B$ are parts of alternative causes of $C$ while $A, B$, and $D$ are contained in alternative causes of $E$. The two causal structures possibly underlying the list in Table 4 are graphed in Fig. 1.

Step 7 of CNA reflects that ambiguity by assigning the following two alternative complex minimal theories to the list of Table 4.

(13) $\left(A X_{1} \vee B X_{2} \vee Y_{C} \Rightarrow C\right) \wedge\left(C X_{3} \vee D X_{4} \vee Y_{E} \Rightarrow E\right)$

(14) $\left(A X_{1} \vee B X_{2} \vee Y_{C} \Rightarrow C\right) \wedge\left(A X_{5} \vee B X_{6} \vee D X_{4} \vee Y_{E} \Rightarrow E\right)$

Based on the list of Table 4 alone it is not determinable whether the behavior of $A, B, C, D, E$ is regulated by a chain or a common cause structure. If no prior causal knowledge is available that disambiguates the inference, a disambiguation has to await later expansions of the factor frame and a pertinent collection of further data. If it is, for instance, found that by manipulating a further factor $F$ it is possible to manipulate $C$ while $E$ remains unchanged, the structure behind Table 4 can unambiguously be identified as a common cause structure. I systematically investigate the ambiguities that may arise in the course of uncovering deterministic causal chains in Baumgartner (2008b). 
Before we move on to consider the consequences of violations of (PEX), emphasis must be put on a major difference between Ragin's $Q C A$-algorithm and CNA that is exhibited by this second exemplary application of CNA. An application of the $Q C A$-algorithm presupposes that factors (or variables) that function as causes in an investigated structure are independent and are, thus, co-instantiable in all logically possible combinations. ${ }^{20}$ This assumption allows for a recourse to the well-known Quine-McCluskey optimization of truth functions in order to minimalize sufficient conditions within the $Q C A$ framework. ${ }^{21}$ This independence assumption, however, has the considerable drawback that causal chains cannot be analyzed by means of $Q C A$, for chains involve dependencies among cause factors. As soon as the independence assumption is dropped-as done in the context of CNA-Quine-McCluskey optimization no longer eliminates all redundancies. The exemplary coincidence list in Table 4 features a dependency among $A \vee B$ and $C$ which all could function as causes within the underlying structure. There is no row in Table 4 reporting a coincidence of, say, $A$ and $\bar{C}$. Quine-McCluskey optimization only eliminates redundant conjuncts of sufficient conditions if a respective truth table contains two rows which differ only with respect to presence and absence of that conjunct. Thus, minimalizing the sufficient conditions of $E$ in Table 4 along the lines of Quine-McCluskey would not identify, say, $A$ as a minimally sufficient condition of $E$, notwithstanding the fact that Table 4 does not contain a coincidence of $A$ and $\bar{E}$. Rendering coincidence lists generated by causal chains amenable to a Boolean analysis, accordingly, calls for a custom-built minimalization procedure that differs from a standard Quine-McCluskey optimization insofar as it systematically tests conjuncts $Z_{i}$ of a sufficient condition $X_{i}$ for eliminability, irrespective of whether the corresponding coincidence list contains another sufficient condition $X_{j}$ that only differs from $X_{i}$ with respect to presence and absence of $Z_{i}$.

\section{Empirical exhaustiveness violated}

As indicated in Sect. 4, assuming the exhaustiveness of analyzed data (PEX) is a precondition of an unambiguous inference to a deterministic structure. Nonetheless, (PEX) is not a necessary assumption on which an application of CNA must be based, for even inexhaustive data provides some information as to underlying causal structures. In order to illustrate this, consider the four coincidences listed in Table 5 which are all likewise contained in Tables 3 and 4 . Against the assumably homogeneous backgrounds of the coincidences in Table $5 A$ and $B$ are each minimally sufficient for the other three factors, while the dependencies among $C, D$, and $E$ are symmetric and, thus, not causally interpretable. Accordingly, CNA assigns the following complex minimal theory to Table 5:

\footnotetext{
${ }^{20}$ May's four-field method (cf. May 1999) also requires potential causes of an investigated effect to be independent in this sense. Structures featuring dependencies among causes such as causal chains, hence, can neither be directly analyzed by $Q C A$ nor four-field testing. For a discussion of the limitations of the four-field method when it comes to uncovering chains cf. Baumgartner and Graßhoff (2004, Chap. 12).

${ }^{21}$ Cf. Quine (1952, 1959), Ragin (1987).
} 
Table 5 An exemplary inexhaustive coincidence list

\begin{tabular}{lllll}
\hline$A$ & $B$ & $C$ & $D$ & $E$ \\
\hline 1 & 1 & 1 & 1 & 1 \\
1 & 0 & 1 & 1 & 1 \\
0 & 1 & 1 & 1 & 1 \\
0 & 0 & 0 & 0 & 0 \\
\hline
\end{tabular}

$\left(A X_{1} \vee B X_{2} \vee Y_{C} \Rightarrow C\right) \wedge\left(A X_{3} \vee B X_{4} \vee Y_{D} \Rightarrow D\right) \wedge\left(A X_{5} \vee B X_{6} \vee Y_{E} \Rightarrow E\right)$

If (PEX) is assumed to be satisfied, (15) constitutes CNA's final output. Yet, if (PEX) is not taken for granted, subsequent extensions of the list in Table 5 are possible. Additional coincidences, of course, may drastically change CNA's output. Depending on whether Table 5 is complemented in terms of, say, Tables 3 or 4 , CNA determines the structure underlying an accordingly complemented list to be the result of a common cause structure or a chain, respectively. In both cases, $A$ and $B$ are no longer held to be causally relevant to $D$. Nonetheless, the causal relevance of $A$ and $B$ to $C$ and $E$ is untouched by extending Table 5 in the sense of either Table 3 or 4 .

Table 5 only features four of the 32 logically possible coincidences over the frame $\{A, B, C, D, E\}$. If (PEX) is not taken for granted, any of the 28 remaining coincidences may be observed later on and integrated into Table 5. However, only a small subset of all these logically possible extensions would be causally interpretable. Suppose, for instance, that all 28 remaining coincidences are in fact incorporated in Table 5. The result is a complete coincidence list, which, as shown in Sect.3, does not feature any deterministic dependencies. The same consequence follows from extending Table 5 in terms of lists (a) or (b) in Table 6. Neither (a) nor (b) are causally interpretable because none of the involved factors can be seen as an effect of an underlying structure. For all factors there is a pair of rows, such that the corresponding factor is the only varying factor in that pair. ${ }^{22}$ Thus, lists (a) and (b) are inhomogeneous and, accordingly, $\mathrm{W}=\emptyset$. An extension of Table 5 as indicated in (c), on the other hand, does not altogether resist a causal interpretation. $A$ and $B$ are still minimally sufficient for $C, D$, and $E$, yet factor $D$ cannot be an effect of the underlying structure any longer. The newly added coincidence features $D$ as the only varying factor when compared to the last coincidence listed in (c). Thus, (c) is not homogeneous with respect to $D . D$ cannot be integrated into an underlying structure as root factor either, for it is not part of a minimally sufficient condition of any of the possible effects contained in (c). In consequence, CNA assigns a minimal theory to (c) that corresponds to (15) reduced by the middle conjunct. In the same vein, extensions of Table 5 may be inhomogeneous in regard to any other effects in (15).

These examples of violated empirical exhaustiveness demonstrate that causal reasoning based on insufficient data is radically underdetermined. Nonetheless, inexhaustive lists allow for excluding some causal structures from possibly underlying a respective list. For example, no extension of the list in Table 5 will ever reveal $D$ to be a cause of either $A$ or $B$. The assumed homogeneity of Table 5 determines that,

\footnotetext{
22 Cf. Sect. 4.
} 
Table 6 (a) and (b) are two extensions of Table 5 that are not causally interpretable; (c) does not allow for an integration of $D$ into the underlying causal structure

Added coincidences are marked with "+"

\begin{tabular}{rrrrr}
\hline$A$ & $B$ & $C$ & $D$ & $E$ \\
\hline 1 & 1 & 1 & 1 & 1 \\
+1 & 1 & 0 & 1 & 1 \\
+1 & 1 & 1 & 0 & 1 \\
+1 & 1 & 1 & 1 & 0 \\
1 & 0 & 1 & 1 & 1 \\
0 & 1 & 1 & 1 & 1 \\
0 & 0 & 0 & 0 & 0 \\
\hline
\end{tabular}

(a)

\begin{tabular}{rllll}
\hline$A$ & $B$ & $C$ & $D$ & $E$ \\
\hline 1 & 1 & 1 & 1 & 1 \\
1 & 0 & 1 & 1 & 1 \\
0 & 1 & 1 & 1 & 1 \\
+0 & 0 & 0 & 0 & 1 \\
+0 & 0 & 0 & 1 & 0 \\
+0 & 0 & 1 & 0 & 0 \\
0 & 0 & 0 & 0 & 0 \\
\hline
\end{tabular}

(b)

\begin{tabular}{rllll}
$A$ & $B$ & $C$ & $D$ & $E$ \\
\hline 1 & 1 & 1 & 1 & 1 \\
1 & 0 & 1 & 1 & 1 \\
0 & 1 & 1 & 1 & 1 \\
+0 & 0 & 0 & 1 & 0 \\
0 & 0 & 0 & 0 & 0 \\
\hline
\end{tabular}

(c)

even though $A$ and $B$ may or may not be contained in an underlying causal structure, if they are thus contained, they are root factors of that structure. For both $A$ and $B$ there is a pair of rows in Table 5 such that they are the only varying factors in that pair, and, as upon extending coincidence lists no coincidences are removed, all extensions of Table 5 will be inhomogeneous with respect to $A$ and $B$. Accordingly, CNA can be said to identify all causal structures not featuring causal relevance of either $C, D$, or $E$ to $A$ and $B$ as possibly underlying the coincidences in Table 5. That set of causal structures also includes the empty structure, i.e. the structure such that $A, B, C, D$, and $E$ are mutually causally independent.

Depending on the previous causal knowledge about the structure under investigation the amount of possible extensions of a given coincidence list may be narrowed down significantly. Certain causal reasoning methodologies available in the literature, hence, propose to supplement inexhaustive data by assumptions embedded in the available causal knowledge about the examined process. ${ }^{23}$ Thus, the underdetermination of causal reasoning based on inexhaustive data may be compensated by additional causal assumptions. Or put differently, the amount of elements in the set of structures assigned to an inexhaustive list as shown in Table 5 can be reduced if it is e.g. known beforehand that certain factors cannot be causally related or that some factor can only be the effect and not the cause of some other factor. However, whenever such previous causal knowledge is not available, inexhaustive empirical data inevitably underdetermines causal inferences.

\section{Summary}

The causal structures and the data analyzed in this paper all violate the causal faithfulness assumption and, accordingly, are not processable by standard algorithms analyzing causal Bayesian networks as presented e.g. in Spirtes et al. (2000). The procedure developed in this paper renders deterministic causal structures mechanically analyzable notwithstanding the fact that they violate faithfulness. CNA implements Boolean techniques and is custom-built for deterministic structures. Contrary to other algorithms embedded in the Boolean tradition as Ragin's $Q C A$-algorithm, CNA does not presuppose that factors operating as causes in an investigated structure are indepen-

$\overline{23 \text { Cf. e.g. Ragin (1987, Chap. 7 }}$; 2000, pp. 139-141, 198-202, 300-308). 
dent. Dropping that independence assumption, on the one hand, renders CNA capable of analyzing chainlike structures, on the other hand, prevents CNA from simply drawing on standard Quine-McCluskey optimization when it comes to minimalizing deterministic dependencies.

Sections 3 and 4 have shown that not every coincidence list is causally analyzable. Moreover, in Sect. 9 we have seen that not all coincidence lists can be unambiguously identified to be the result of one specific causal structure. Like algorithms for causal Bayes nets, CNA sometimes assigns multiple structures to corresponding data. Accordingly, CNA cannot be seen as a complete inference procedure in the sense that it assigns a specific deterministic structure to a coincidence list whenever the coincidences in that list are in fact the result of such a structure. Empirical data may be insufficient to unambiguously uncover its causal regularities. However, the claim defended in this paper is that CNA is a correct causal inference procedure in the sense that whenever CNA assigns a set containing one or more deterministic structure(s) to a homogenous coincidence list, that list is in fact generated by a member of that set.

Acknowledgements This paper is greatly indebted to Timm Lampert and Daniel Engler, in collaboration with whom first attempts at adapting Quine-McCluskey optimization for the discovery of complex causal structures have been made. Moreover, I thank Clark Glymour, Manuel Jakob, Tim Raez, Charles Ragin and two anonymous reviewers for this journal for very valuable comments on earlier drafts. Finally, I am grateful to the Swiss National Science Foundation for generous support of this work (grant 1114-066803.01/1).

\section{References}

Baumgartner, M. (2006). Complex causal structures. Extensions of a regularity theory of causation. Ph.D. thesis, University of Bern, Bern.

Baumgartner, M. (2008a). Regularity theories reassessed. Philosophia. http://www.springerlink.com/ content/j2666001q3258005/.

Baumgartner, M. (2008b). The casual chain problem.

Baumgartner, M., \& Graßhoff, G. (2004). Kausalität und kausales Schliessen. Eine Einführung mit interaktiven Übungen. Bern: Bern Studies.

Ehring, D. (1982). Causal asymmetry. The Journal of Philosophy, 79, 761-774.

Glymour, C. (1997). A review of recent work on the foundations of causal inference. In V. R. McKim \& S. P. Turner (Eds.), Causality in crisis? (pp. 201-248). Notre Dame: University of Notre Dame Press.

Glymour, C. (2007). Learning the structure of deterministic systems. In A. Gopnick \& L. Schulz (Eds.), Causal learning. Psychology, philosophy, and computation (pp. 231-240). New York: Oxford University Press.

Graßhoff, G., \& May, M. (2001). Causal regularities. In W. Spohn, M. Ledwig, \& M. Esfeld (Eds.), Current issues in causation (pp. 85-114). Paderborn: Mentis.

Hausman, D. (1998). Causal asymmetries. Cambridge: Cambridge University Press.

Lemmon, E. J. (1965/1978). Beginning logic. Indianapolis: Hackett.

Mackie, J. L. (1974). The cement of the universe. A study of causation. Oxford: Clarendon Press.

May, M. (1996). Experimentation and the formation of causal theories. In Workshop on cognitive modeling '96. http://citeseer.ist.psu.edu/330253.html.

May, M. (1999). Kausales Schliessen. Eine Untersuchung über kausale Erklärungen und Theorienbildung. Ph.D. thesis, Universität Hamburg, Hamburg.

Mirsalehi, M. M., \& Gaylord, T. K. (1986). Logical minimization of mulitlevel coded functions. Applied Optics, 25, 3078-3088.

Pearl, J. (1985). Bayesian networks: A model of self-activated memory for evidential reasoning. In Proceedings of the 7th Conference of the Cognitive Science Society (pp. 329-334). Irvine: University of California. 
Quine, W. v. O. (1952). The problem of simplifying truth functions. The American Mathematical Monthly, $59,521-531$.

Quine, W. v. O. (1959). On cores and prime implicants of truth functions. American Mathematical Monthly, $66,755-760$.

Ragin, C. C. (1987). The comparative method. Berkeley: University of California Press.

Ragin, C. C. (2000). Fuzzy-set social science. Chicago: University of Chicago Press.

Sanford, D. (1976). The direction of causation and the direction of conditionship. The Journal of Philosophy, 73, 193-207.

Sasao, T. (1999). Switching theory for logic synthesis. Boston: Kluwer.

Spirtes, P., Glymour, C., \& Scheines, R. (1993/2000). Causation, prediction, and search (2nd ed.). Cambridge: MIT Press. 